\title{
The Status of Oxygen Abundances in Metal-Poor Stars
}

\author{
Ruth C. Peterson \\ Astrophysical Advances and UCO/Lick Observatories
}

\begin{abstract}
The sensitivity of various methods of oxygen-abundance determination to the structure of the model atmosphere in a classical analysis is described, with emphasis on the treatment of convection. Oxygenabundance results presented during this meeting are outlined. Because the oxygen diagnostics are sensitive in different ways to the choice of model stellar parameters and to the treatment of convection, it is not surprising that different methods applied by different groups give different results. Most are consistent with an increase in $[\mathrm{O} / \mathrm{Fe}]$ as $[\mathrm{Fe} / \mathrm{H}]$ declines, but the slope is uncertain still, and the scatter is large at very low abundances. The trend may continue to low $[\mathrm{Fe} / \mathrm{H}]$, but more stars and more checks are needed.
\end{abstract}

\section{Classical Abundance Analyses and the Treatment of Convection}

In a classical abundance analysis, a one-dimensional, static model atmosphere in local thermodynamic equilibrium (LTE) is adopted. The very simple mixinglength approximation is invoked to model convection, which accounts for flux transport in stars cooler than $9000 \mathrm{~K}$ at and below the point in the stellar photosphere where the atmosphere is becoming optically thin. In real stars, departures from LTE may occur (e.g. Thévenin \& Idiart 1999), and convection is much more complex, with 3D flows. Talks in these proceedings by Kiselman (JD8.005T) and by Asplund (JD8.008T) describe progress in treating these phenomena, while effects of 2D inhomogeneities are discussed by Cayrel et al. (JD8.004T). Straightening this out is important, given the impact of the oxygen abundance on diverse problems, as discussed for LiBeB by Cassé (JD8.003T), for chemical evolution of the Galaxy by Matteucci (JD8.009T), and for globular-cluster ages by VandenBerg et al. (JD8.014T). For a summary see Lambert (JD8.006T).

Within the classical framework, convection may be treated in different ways. Castelli, Gratton, \& Kurucz (1997) have investigated the effect of the treatment of convection in Kurucz $(1993,1994,1995)$ models, in which the convective flux is allowed to "overshoot" its nominal cessation point by one mixing length. Figs. 11 and 12 of Castelli et al. show that turning off overshoot changes the shape of the temperature distribution of the classical model at just the point where the photosphere is becoming optically thin in the visible wavelength region. Thus the classical analysis of any line or color formed in this region will be sensitive to whether convective overshoot is turned on, as in the Kurucz $(1993,1994,1995)$ models, or turned off, as in models Castelli et al. calculated explicitly. (Both sets of models are available from the Kurucz web site at http://cfaku5.harvard.edu.) 
For the Sun, Castelli et al. find better agreement in both colors and Balmer-line profiles when this overshoot option is turned off.

\section{The Model Dependence of Oxygen Abundance Diagnostics}

Oxygen-abundance diagnostics generally rely on weak lines of higher-than-average temperature sensitivity. Consequently the choice of convective overshoot affects the deduced oxygen abundance directly, by dictating the temperature and the temperature gradient of the line-forming region, and indirectly, in cases where colors are used to establish the stellar effective temperature $T_{\text {eff }}$.

Four diagnostics of the oxygen abundance are widely used: the UV OH features found from $2900-3180 \AA$, the IR-OH lines near $1.6 \mu \mathrm{m}$, the [O I] forbidden lines at $6300.3 \AA$ and $6363.6 \AA$, and the O I permitted near-IR triplet at 7771 $7775 \AA$. Because they derive from molecules that are easily dissociated, the UV$\mathrm{OH}$ and IR-OH lines all decrease in strength very rapidly as temperature rises. The [O I] line is the ground state of the primary ionization stage of its element, so it decreases somewhat more rapidly with temperature than do most $\mathrm{Fe} I$ lines, which at $\sim 3 \mathrm{eV}$ are at higher excitation. The $\mathrm{O}$ I near-IR line arises from a very highly excited level, $\sim 8 \mathrm{eV}$, so it actually increases in strength as temperature rises. Again due to dissociation, the molecular $\mathrm{OH}$ features decrease in strength as gravity decreases, while the $[\mathrm{O} \mathrm{I}]$ and $\mathrm{O}$ I near-IR atomic lines do the opposite.

Because of their different wavelengths, the various oxygen line diagnostics are formed at different physical depths in a stellar photosphere. This is based on the wavelength dependence of the continuous opacity, plotted for the Sun in Figs. 8.6a and 8.7 of Gray (1992). Peaking at $\sim 9000 \AA$, the opacity goes through a minimum at $1.6 \mu \mathrm{m}$, and also decreases towards the UV but with jumps due to Balmer-line absorption and to absorption by metals. As a result, IR-OH lines are formed very much deeper, and UV-OH somewhat deeper, than optical lines.

The bottom line is that differences in oxygen abundance found from different diagnostics by different groups in the same star are likely, unless the model temperature, gravity, and treatment of convection are all appropriately modelled. This is over and above the common difficulty of establishing $\log g f$ values for the various transitions on a consistent scale. Progress on this problem for $\mathrm{UV}-\mathrm{OH}$ is reviewed in these proceedings by Bessell (JD8.001T).

\section{Recent Results for Oxygen Abundances}

Throughout this meeting, many new results have been presented for oxygen abundances. Talks appearing in these proceedings include those by Balachandran (JD8.013T) for IR-OH, Sneden (JD8.012T) for [O I], and García López (JD8.010T) and Boesgaard (JD8.002T) for UV-OH, the latter works including very low-metallicity stars. While some UV-OH oxygen abundances tend to be higher than those from IR-OH lines, this is not evident in our own recent UV-OH work (Peterson, Dorman \& Rood 2000).

Even more new results appearing as posters are found in New Astronomy Reviews. Primas et al. (JD8.016P) have derived abundances from the near-IR O I triplet in 35 stars with $-2.5 \leq[\mathrm{Fe} / \mathrm{H}] \leq 0.0$. Cunha et al. (JD8.017P) have 
studied $[\mathrm{OI}]$ and $\mathrm{O} \mathrm{I}$ in $\mathrm{F}$ and $\mathrm{G}$ dwarfs with $-0.75 \leq[\mathrm{Fe} / \mathrm{H}] \leq+0.15$. Melendez et al. (JD8.020P) have examined IR-OH in 14 stars with $-2.2 \leq[\mathrm{Fe} / \mathrm{H}] \leq-0.75$. Nissen et al. (JD8.021P) analyzed [O I] in 15 dwarfs and subgiants with $-1.8 \leq$ $[\mathrm{Fe} / \mathrm{H}] \leq-0.7$. Takada-Hidai et al. (JD8.023P) scrutinized [O I] and $\mathrm{O} \mathrm{I}$ in six giants and one dwarf.

Virtually all results show the oxygen abundance rising as stellar metallicity declines. Unfortunately, the number of analyses for extremely metal-poor stars is quite small, and the scatter is large. More stars need to be analyzed in more different ways and with more thorough cross-checks to confirm the possible rise in the oxygen abundance below $[\mathrm{Fe} / \mathrm{H}]<-2.5$ so important to many areas.

\section{Suggestions for Future Work}

In light of the above, suggestions are made for future analyses. Given the substantial advances made recently, considerable future progress is to be expected.

- Include several standard stars observed by other groups, such as the metalpoor turnoff stars HD 19445, HD 84937, and HD 140283 and the cooler stars HD 103095 and $B D+3740$. Without stars in common, it is impossible to compare results from different efforts.

- List $T_{\text {eff }}, \log g$, and $[\mathrm{Fe} / \mathrm{H}]$ values adopted for each star along with equivalent widths (where available) and $\log g f$ values. In UV and IR investigations, compare $[\mathrm{Fe} / \mathrm{H}]$ values as determined from the relevant wavelength region to those from optical spectra using the same models.

- Test the sensitivity of the results to the treatment of convection.

- Where continuum placement is an issue, plot fits to the spectral regions adjacent to those of the features of interest.

- To validate model dependencies, analyze species of similar formation characteristics - such as blue $\mathrm{CH}$ at $4300 \AA$ and UV CH in addition to UV OH, and other high-excitation atomic lines along with the near-IR O I triplet.

\section{References}

Castelli, F., Gratton, R. G., \& Kurucz, R. L. 1997, A\&A, 318, 841

Gray, D. F. 1992, The Observation and Analysis of Stellar Photospheres (Cambridge: Cambridge Univ. Press)

Kurucz, R. L. 1993, ATLAS9 Stellar Atmosphere Programs and $2 \mathrm{~km} \mathrm{~s}^{-1}$ Grid (Kurucz CD-ROM No. 13)

Kurucz, R. L. 1994, Solar Abundance Model Atmospheres of 0,1,2,4,8 km s s $^{-1}$ (Kurucz CD-ROM No. 19)

Kurucz, R. L. 1995, updated model grids posted at http://cfaku5.harvard.edu

Peterson, R. C., Dorman, B., \& Rood, R. T. 2000, ApJ, submitted (astro$\mathrm{ph} / 0009208)$, and in preparation

Thévenin, F., \& Idiart, T. P. 1999, ApJ, 521, 753 